\title{
Carbon-containing High Temperature Piezoresistive Materials
}

\author{
E. Ionescu ${ }^{1}$, H.-J. Kleebe ${ }^{1}$, K. Krause ${ }^{1}$, N. Nicoloso ${ }^{1}$, L. Toma ${ }^{2}$, and R. Riedel ${ }^{1}$ \\ ${ }^{1}$ Technische Universität Darmstadt, Fachbereich Material- und Geowissenschaften, Petersenstrasse \\ 32, 64287 Darmstadt, Germany \\ ${ }^{2}$ Fraunhofer-Institut für Silicatforschung ISC, Gottlieb-Keim-Str. 60, 95448 Bayreuth, Germany
}

\begin{abstract}
Silicon oxycarbide-based ceramics (SiOC) with contents of segregated carbon in the range from 9 to $33 \mathrm{vol} \%$ have been synthesized by pyrolysis of polysiloxanes $\left(1100^{\circ} \mathrm{C}\right)$ and subsequent annealing at high temperatures $\left(1400-1600{ }^{\circ} \mathrm{C}\right)$. The samples annealed at 1400 and $1600{ }^{\circ} \mathrm{C}$ were found to exhibit piezoresistivity with room temperature gauge factors $10^{2}<\mathrm{k}<3 \times 10^{3}$, two to three orders of magnitude higher than those of conventional pressure sensors; whereas the sample synthesized at $1100{ }^{\circ} \mathrm{C}$ did not show any piezoresistive effect. X-ray and TEM-analysis of the prepared materials indicate that the piezoresistive behaviour strongly depends on the microstructure of the compounds, most likely . on the presence and nature of the segregated carbon phase. The thermal stability and microstructure of the carbon phase has been assessed by means of temperature-dependent Raman spectroscopy $\left(25<\mathrm{T}<600{ }^{\circ} \mathrm{C}\right)$. Within the investigated temperature range the microstructure of the carbon phase is essentially unaltered, suggesting that the piezoresistive effect seen at RT will persist up to high temperatures, i.e. up to the thermal stability limit of $\operatorname{SiOC}\left(T \approx 1400{ }^{\circ} \mathrm{C}\right)$. Due to their other outstanding properties, e.g. thermal and chemical stability as well as excellent creep resistance, the $\mathrm{SiOC}$ compounds allow a substantial extension of the operation pressure and temperature range of piezoresistive sensors beyond the current state-of-the-art.
\end{abstract}

Key words: piezoresistive sensor, nanocomposites, nano-carbon, Raman spectroscopy, polymer-derived ceramics (PDCs)

\section{Introduction}

To date resistive, piezoresistive and capacitive measuring principles are predominantly used for industrial pressure sensors, with an increasing need for sensors based on the piezoresistive effect. The main reasons are the high sensitivity, reproducibility, long term stability and robustness of the sensing resistor against interfering factors. Piezoresistive sensors are usually made of doped semiconducting materials or polymer composites [1]. However, these materials are not applicable at elevated temperature or in aggressive environment. On the other hand most of the high-temperature and corrosion stable materials do not exhibit useful piezoresistivity [2]. Polymer derived ceramics (PDCs) are materials which have been intensively studied in the last three decades. They describe a new class of ceramics which can be prepared without any additives by thermal handling of appropriate precursors in inert or reactive atmosphere.
Polymer derived ceramics are amorphous materials and were shown to keep their amorphous nature up to temperatures ranging from 1000 to $1800{ }^{\circ} \mathrm{C}$, depending on their chemical composition and structure. Recently, a topological model for silicon oxycarbides has been proposed, which assumes that SiOC consists of two interpenetrating $\mathrm{SiO}_{2}$ and Cbased networks $[3,4,5]$. In addition, the microstructure of $\mathrm{SiOC}$ exhibits a finely dispersed carbon phase. The amount and nature of the segregated carbon within the microstructure of PDCs has a crucial effect on their properties (electrical conductivity, hightemperature creep behavior, devitrification and crystallization behavior).

Polymer-derived ceramics (PDCs) have recently been shown to be promising piezoresistive materials with large gauge factors $[6,7,8,9]$. As required for high temperature sensor applications, they have excellent stability at elevated temperature and in harsh conditions $[10,11,12]$. However, 
PDCs not only exhibit unusual structural properties. A variety of functionalities can be easily introduced, such as electrical conductivity, photo luminescence or magnetic and catalytic properties [10, 11, 12] Furthermore, PDCs have the advantage that they can be processed like thermoplastic materials to obtain complex three-dimensional shapes. The polymer-derived route provides high purity compounds with very good homogeneity as a prerequisite for industrial application. In the following we present experimental evidence that these materials are promising candidates for a new class of pressure sensors showing a strong piezoresistive effect which relies on the formation of a short range-ordered carbon network.

\section{Experimental}

A commercially available poly(methylsilsesquioxane) (PMS MK, Wacker Chemie AG, München, Germany) was used for the preparation of the silicon oxycarbide samples. Zirconium acetylacetonate served as crosslinking agent. A batch of the preceramic polymer was cross-linked at $250{ }^{\circ} \mathrm{C}$ for $30 \mathrm{~min}$, ball milled, and finally sieved to a particle size of $100 \mu \mathrm{m}$. Subsequently, the ground powder was pyrolyzed in flowing argon at $900{ }^{\circ} \mathrm{C}$ for 30 min. The pyrolyzed SiOC powder (denoted as MK_900) was mixed with the catalytically treated and cross-linked polymer (75 wt\% SiOC powder $+25 \mathrm{wt} \%$ MK polymer) by ball milling and finally sieved to a particle size of $100 \mu \mathrm{m}$. Shaping of the polymer-powder mixture was achieved by uniaxial warm pressing at $25 \mathrm{MPa}$ and $180^{\circ} \mathrm{C}$. Subsequently, the bulk sample was pyrolyzed in a tube furnace up to the pyrolysis temperature of $1100{ }^{\circ} \mathrm{C}$ and a 5 -h holding time (sample denoted as MK_1100). Further annealing treatment of the initial MK_1100 sample was performed at $1400{ }^{\circ} \mathrm{C}$ for $5 \mathrm{~h}$ under argon atmosphere (sample denoted as MK_1400) [7].

For the preparation of a carbon-rich sample, the polymer PMS MK was blended with back carbon (83.5 wt\% MK polymer + 16.5 wt\% carbon black) and subsequently cross-linked at $250{ }^{\circ} \mathrm{C}$ and pyrolyzed at $900{ }^{\circ} \mathrm{C}$ in argon atmosphere (sample denoted as C_900).

The ceramic powders MK_900 and C_900 were hot pressed in argon at $1600{ }^{\circ} \mathrm{C}(3 \overline{0} \mathrm{MPa})$ to dense monoliths denoted as MK_1600 and C_1600 (Figure 1) [5, 13].

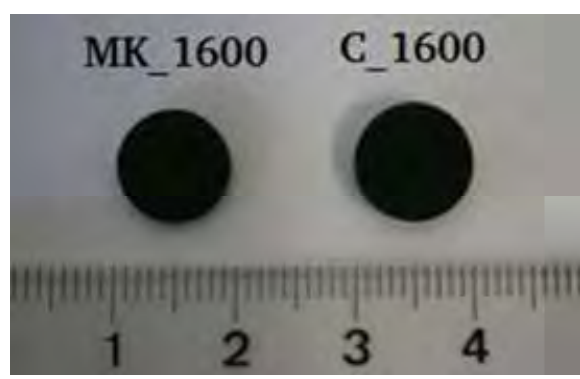

Fig. 1. Photograph of the SiOC samples prepared upon hot-pressing technique [13].

Elemental analysis of the samples was performed by hot gas extraction (Mikrolabor Pascher, Remagen, Germany).

Transmission electron microscopy (TEM) imaging was performed using a CM20STEM instrument (FEl, Eindhoven, The Netherlands) operating at $200 \mathrm{kV}$ on TEM-foils obtained from the bulk samples.

Micro-Raman spectra (10 scans, each scan lasting $3 \mathrm{~s}$ ) were recorded with a Horiba HR800 micro-Raman spectrometer (Horiba Jobin Yvon, Bensheim, Germany) equipped with an Ar laser (wavelength $514.5 \mathrm{~nm}$ ). The excitation line has its own interference filter (to filter out the plasma emission) and a Raman notch filter (for laser light rejection). The measurements were performed by using a grating of $1800 \mathrm{gmm}-1$ and a confocal microscope (magnification 100×, NA 0.5) with a $100 \mu \mathrm{m}$ aperture, giving a resolution of 2-4 $\mu \mathrm{m}$. The laser power $(20 \mathrm{~mW})$ was attenuated by using neutral density (ND) filters; thus, the power on the sample was in the range of $2 \mathrm{~mW}-20 \mu \mathrm{W}$.

\section{Results and discussion}

In the following we report on structural and spectroscopic investigations of the SiOC ceramic samples to get further information on the relationship between their microstructure and the piezoresistive behavior. The crystalline phase composition and the microstructure of the samples was studied by X-ray diffraction (XRD) and transmission electron microscopy (TEM). Furthermore, Raman spectroscopy was chosen to probe the local bonding in the segregated carbon phase.

\section{XRD and TEM of piezoresistive SiOC}

Fig. 2 presents the XRD spectra of the SiOC compounds synthesized at $1100<\mathrm{T}<1600{ }^{\circ} \mathrm{C}$. The data indicate that they are X-ray amorphous (independent of the preparation technique and temperature). 


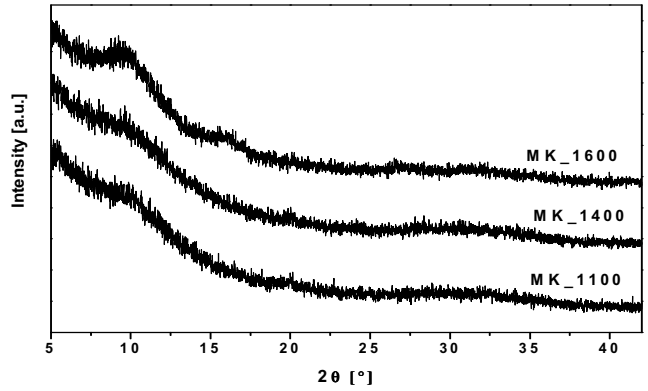

Fig. 2: XRD spectra of SiOC samples prepared at 1100,1400 and $1600{ }^{\circ} \mathrm{C}$.

TEM measurements provide detailed information on the morphology and microstructure of the SiOC samples. In accord with previous investigations [3, 4, 5], glassy silica nano-domains can be identified as well as amorphous/turbostratic carbon which is introduced within the microstructure of SiOC during the heat treatment of the polysiloxane precursor. The segregated carbon phase is found to partially crystallize within the Si-O-C matrix upon high-temperature treatment and can be described as nano-sized turbostratic graphite particles with $\mathrm{d}$ « $10 \mathrm{~nm}$. Fig. 3 presents a TEM image of the MK 1400 sample. The sample is mesoporous and pores are filled with carbon [9]. In contrast to this, no carbonfilled pores were observed in MK_1100. Hence, both samples differ considerably in their content of highly conductive graphitic domains and in case of the MK_1100 sample the carbon network appears to be less pronounced. The carbon inside the pores is expected to build up a 3D-network which will render the material conductive beyond the percolation threshold.

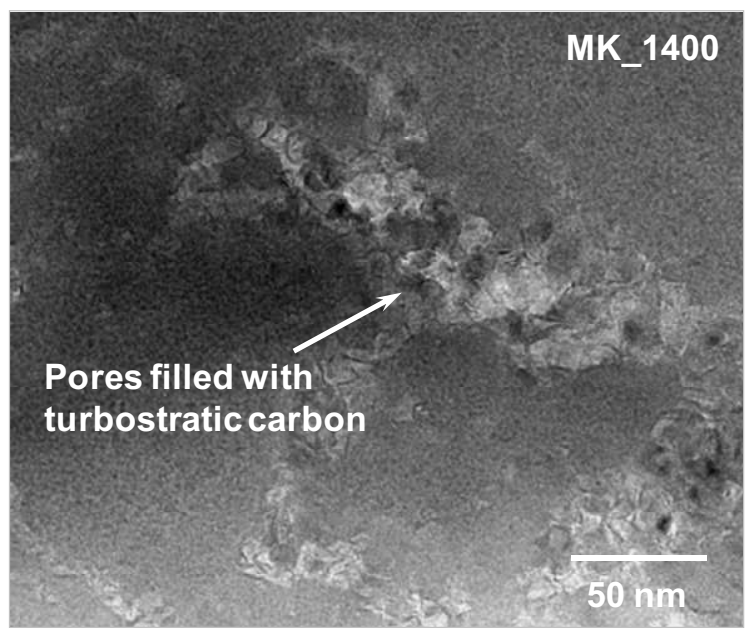

Fig. 3: TEM of SiOC pyrolyzed a $1400^{\circ} \mathrm{C}$. Pores are filled with turbostratic carbon (marked by an arrow) [9] (with permission Wiley-Blackwell).

As shown in Fig. 4 the MK_1400 sample shows piezoresistive behaviour [7]: the resistivity dynamically follows the applied stress. The gauge factor defined as

$$
k \approx \frac{Y}{R_{0}} \frac{\Delta R}{\Delta \sigma}
$$

has been determined to be 145. Here $Y$ denotes the Youngs' modulus (85 GPa for SiOC [7]), $R_{o}$ the resistivity of the stress-free sample and $\Delta R / \Delta \sigma$ the change of the sample resistivity with the applied stress.

Unlike MK_1400, the MK_1100 sample does not show any piezoresistivity, suggesting that the piezoresistive effect is intimately connected with a well-established conductive carbon part (or a silica-carbon interface) within the microstructure of SiOC ceramic.

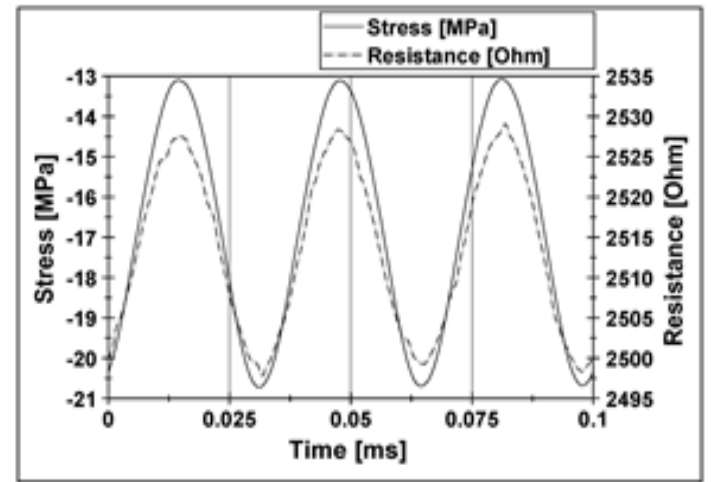

Fig. 4: Room temperature dynamic test of piezoresistivity in the SiOC ceramic MK_1400 [7] (with permission Wiley-Blackwell).

Apart from dynamic tests, the piezoresistive effect of SiOC compounds has been investigated with the use of an uniaxial hot press modified to allow resistance measurements at a pressure of about $15 \mathrm{MPa}$. Table 1 lists the resistance $R_{0}$ of stress-free samples the change of $R$ with applied stress $(\Delta R, \Delta \sigma)$ and the calculated gauge factor $k$ for three SiOC samples deliberately chosen to demonstrate the range of achievable k-values.

Table 1: Electrical resistivity and gauge factors of carbon containing PDCs demonstrating the accessibility of gauge factors $10^{2}<k<10^{3}$ (at RT).

\begin{tabular}{|c|c|c|c|c|}
\hline Sample & $\mathrm{R}_{0}[\Omega]$ & $\begin{array}{c}\Delta \mathrm{R} \\
{[\Omega]}\end{array}$ & $\begin{array}{c}\Delta \sigma \\
{[\mathrm{MPa}]}\end{array}$ & $k$ \\
\hline MK_1400[4] & 3300 & 35 & 8 & 145 \\
\hline C_1600 & 35.7 & 3.64 & 17 & 660 \\
\hline MK_1600 & 548 & 252 & 15.7 & 3230 \\
\hline
\end{tabular}


Temperature dependent Raman investigations of the carbon phase in SiOC

Raman spectroscopy probes the local structure of a compound and many investigations have been carried out to describe short range ordering in different carbon materials, from structurally not well-defined carbon soots to highly ordered material. In perfectly long range ordered carbon (graphene) only the in-plane stretching mode with $E_{2 g}$ symmetry ("G" mode) and its $2^{\text {nd }}$ harmonic ("2D" mode) are raman-active. Looking at the counterpart, disordered (amorphous) carbon, symmetry-forbidden modes like $A_{1 g}$ ("D" mode) become observable and dominate the spectra. In case of the commonly used laser excitation at $514 \mathrm{~nm}$, the $D$ line appears at about $1350 \mathrm{~cm}^{-1}$. The $\mathrm{G}$ mode is non-dispersive and occurs at about $1580 \mathrm{~cm}^{-1}$, independent of the laser wavelength. The intensity ratio of the $D$ and $G$ mode, $I_{D} / I_{G}$, can be used for quality control. The lower its value, the better crystallized the material. Fig. 5 presents the Raman spectrum of the sample MK_1600. Deconvolution of the spectrum reveals a strong $D$ line at $1350 \mathrm{~cm}^{-1}$, a structured $G$ line $\left(G+D^{\prime}\right.$, 1585 and $1620 \mathrm{~cm}^{-1}$ ) and weak signals $D$ " $\left(1500 \mathrm{~cm}^{-1}\right)$ and $T\left(1200 \mathrm{~cm}^{-1}\right)$. The latter two signals have been attributed to $\mathrm{sp}^{3}-\mathrm{sp}^{2}$ carbon bonds and highly disordered graphitic carbon [14]. The $D^{\prime}$ band at $1620 \mathrm{~cm}^{-1}$ can be assigned to an E mode for a graphite "boundary" layer adjacent to an intercalant layer, not sandwiched between two other graphite planes [15]. As is evident from Fig. 5, the carbon phase in SiOC is highly disordered but cannot be considered as completely amorphous since there exists some degree of order in the basal plane (the $G$ line is pronounced and not negligible).

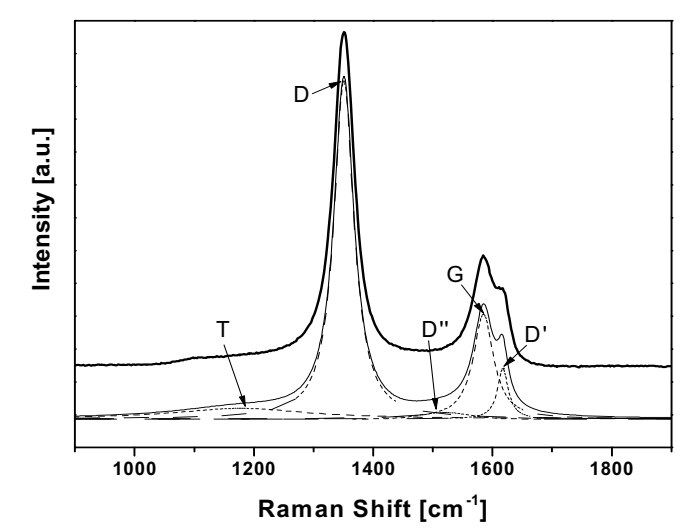

Fig.4: Raman spectrum of MK_1600 (solid line) and its deconvolution providing evidence for ordered $\left(G+D^{\prime}\right)$ and non-crystalline carbon $\left(D, D^{\prime \prime}\right.$ and $\left.T\right)-$ dashed lines [13].

Heating up the SiOC samples in air may cause a strong change in $I_{D} / I_{G}$. The disordered carbon is expected to oxidize more easily than the highly ordered graphitic part and consequently, a change in $I_{D} / l_{G}$ will indicate thermal instability. Within the investigated temperature range (i.e., $25<\mathrm{T}<600{ }^{\circ} \mathrm{C}$ ) no major changes of $\mathrm{I}_{\mathrm{D}} / \mathrm{I}_{\mathrm{G}}$ occur in all of the samples. The carbon microstructure is essentially unaltered and, consequently, the piezoresistive effect observed at room temperature is expected to persist up to elevated temperature, i.e. up to the thermal stability limit of the materials (approximately $1400{ }^{\circ} \mathrm{C}$ ). First high temperature experiments confirm this result, as gauge factors in the range of $10^{2}-10^{3}$ (depending on the temperature and applied stress) can be determined at $800<\mathrm{T}<1400{ }^{\circ} \mathrm{C}$ [16].

To our knowledge, high temperature piezoresistivity has been observed only in one further carbon-containing compound, i.e. $\operatorname{SiCO}(\mathrm{N})$. The published gauge factors $\left(k \approx 1000\right.$ at $\left.800^{\circ} \mathrm{C}\right)$ [8] are in good agreement with our values. The piezoresistive effect in $\mathrm{SiOC}$ and $\mathrm{SiCO}(\mathrm{N})$ should be of comparable size, as seen, because the microstructure of the carbon phase is expected to be rather similar in both systems, . However, further systematic work is necessary to establish a profound understanding of the piezoresistive effect in PDCs, notably its possible dependence on local defects in carbon nano-domains.

\section{References}

[1] Y. Kanda, K. Suzuki, Phys. Rev. B 43, 6754-6756 (1991); doi: 10.1103/PhysRevB.43.6754.

[2] a) J. S. Shor, D. Goldstein, A. D. Kurtz, IEEE Trans. Elect. Devices 40, 1093-1099 (1993); doi: 10.1109/16.214734;

b) T. Toriyama, S. Sugiyama, Appl. Phys. Lett. 81, 2797-2799 (2001); doi: 10.1063/1.1513652.

[3] A. Saha, R. Raj, and D. L. Williamson, J. Am. Ceram. Soc. 89 [7], 2188-2195 (2006); doi: 10.1111/j.1551-2916.2006.00920.x.

[4] S. J. Widgeon, S. Sen, G. Mera, E. Ionescu, R. Riedel, and A. Navrotsky, Chem. Mater. 22, 6221-6228 (2010); doi: 10.1021/cm1021432.

[5] B. Papendorf, E. Ionescu, H.-J. Kleebe, C. Linck, O. Guillon, K. Nonnenmacher, R. Riedel, J. Am. Ceram. Soc. 96 [1], 272-280 (2013); doi: $10.1111 /$ jace.12067.

[6] L. Zhang, Y. Wang, Y. Wei, W. Xu, D. Fang, L. Zhai, K.-C. Lin, and L. An, J. Am. Ceram. Soc., 91 [4] 1346-9 (2008); doi: 10.1111/j.1551-2916.2008.02275.x.

[7] R. Riedel, L. Toma, E. Janssen, J. Nuffer, T. Melz, H. Hanselka, J. Am. Ceram. Soc. 93, 920924 (2010); doi: 10.1111/j.1551-2916.2009.03496.x. 
[8] K. Terauds, P.E. Sanchez-Jimenez, R. Raj, C. Vakifahmetoglu, P. Colombo, J. Eur. Ceram. Soc. 30, 2203-2207 (2010); doi: 10.1016/j.jeurceramsoc.2010.02.024.

[9] L. Toma, H.-J. Kleebe, M. M. Müller, E. Janssen, R. Riedel, T. Melz, H. Hanselka, J. Am. Ceram. Soc. 95, 1056-1061 (2012); doi: 10.1111/j.1551-2916.2011.04944.x.

[10] "Polymer Derived Ceramics: from Nanostructure to Applications", Eds. P. Colombo, R: Riedel, H.J. Kleebe, G. D. Soraru, DesTech Publishing House, 2010.

[11] P. Colombo, G. Mera, R. Riedel, G. D. Soraru, J. Am. Ceram. Soc. 93, 1805-1837 (2010); doi: 10.1111/j.1551-2916.2010.03876.x.
[12] E. lonescu, "Polymer Derived Ceramics", in Ceramics Science and Technology, Wiley- $\mathrm{VCH}$, 2012, p. 457-500.

[13] K. Krause, diploma work, TU Darmstadt 2012.

[14] A. Sadezky , H. Muckenhuber, H. Grothe , R. Niessner , U. Pöschl , Carbon 43 1731-1742 (2005); doi: 10.1016/j.carbon.2005.02.018.

[15] Y. Wang, D. C. Alsmeyer, R. L. McCreery, Chem. Mater. 2, 557-563 (1990); doi: $10.1021 / \mathrm{cm} 00011 \mathrm{a} 018$.

[16] E. Ionescu, N. Nicoloso, O. Guillon, R. Riedel, to be published. 